\title{
Correction to: Synthetic Biology and the Translational Imperative
}

\author{
Raheleh Heidari Feidt ${ }^{1} \cdot$ Marcello Ienca $^{1,2} \cdot$ Bernice Simone Elger $^{1,3}$ • \\ Marc Folcher $^{4}$ (D)
}

Published online: 24 January 2018

(C) Springer Science+Business Media B.V., part of Springer Nature 2018

\section{Correction to: Sci Eng Ethics https://doi.org/10.1007/s11948-017-0011-3}

The author group of above-mentioned review paper was incorrectly published in the online article. It should read:

Raheleh Heidari Feidt · Marcello Ienca · Bernice Simone Elger* · Marc Folcher*

*Share the last authorship.

The original article can be found online at https://doi.org/10.1007/s11948-017-0011-3.

Marc Folcher

marc.folcher@bsse.ethz.ch

Raheleh Heidari Feidt

heidarir@tcd.ie

Marcello Ienca

marcello.ienca@hest.ethz.ch

Bernice Simone Elger

b.elger@unibas.ch

1 Institute for Biomedical Ethics, Universität Basel, Bernoullistrasse 28, 4056 Basel, Switzerland

2 Health Ethics \& Policy Lab, Department of Health Sciences and Technology (D-HEST), ETH Zürich, Auf der Mauer 17, 8001 Zurich, Switzerland

3 Center for Legal Medicine, University of Geneva, Geneva, Switzerland

4 Department of Biosystems Science and Engineering (D-BSSE), ETH Zürich, Mattenstrasse 26, 4058 Basel, Switzerland 